\title{
Hyphenated techniques as tools for speciating biological metals: Metallothionein and metal-binding proteins
}

\author{
K.T. Suzuki \\ Faculty of Pharmaceutical Sciences, Chiba University, Inage, Chiba 263-8522, Japan
}

The development of hyphenated techniques for biomedical applications was reviewed from early HPLC-AAS, to analyze metallothionein, to recent HPLC-ICP MS, to reveal the mechanism underlying the interaction between mercuric ions, selenium and selenoprotein P. Future application to the speciation of biological metals was discussed together with the requirements for improvement of the technique.

lthough metals are minor constituents in the body, some are essential in the normal functioning body and others are highly toxic, indicating their critical biological actions, irrespective of their being beneficial or adverse. As a result, the biological effects of metals on health have been of concern from the viewpoints of nutrients, their biological and physiological functions, their toxicological actions and also the biochemical mechanisms of their functions. The detection and characterization of biological trace metals is the first step to address these concerns. However, detection itself has been a challenging problem in terms of trace analysis with accuracy and precision. Biological and environmental trace metals have been analyzed for a long time only at the concentration level, i.e. it has been a challenge in elemental analysis to elevate the sensitivity from $\%$ to the ppm, ppb and ppt levels, but there has been no focus on their chemical forms, i.e. it has been not extended to the level of elemental speciation.

\section{Speciation of biological metals}

The concept of speciation is now defined as the identification and quantification of species. Species are defined from a functional or operational standpoint, or as a specific chemical form (ligand) or oxidation state, i.e. functional, operational and chemical speciation, respectively [1]. Information about the concentrations of metals without speciation is not sufficient to understand the biological reactions, functions and other biological actions of metals.

Trace analysis itself has been a challenging problem and remains so. However, speciation requires sophisticated techniques for separating metals according to their chemical forms or characteristics, and then quantification of metals in each chemical form [2,3]. Although speciation is quite a new technical term [1], biological trace metals have been sepa- rated and quantified in toxicological studies, for example, to correlate the toxic form of cadmium $(\mathrm{Cd})$ in a target organ with its toxicity with an off-line system, such as separation by open column chromatography and detection by atomic absorption spectrophotometry (AAS), i.e. $\mathrm{Cd}$ in the target organ was analyzed as the non-toxic form bound to metallothionein (MT), and toxic forms bound non-selectively to high molecular weight proteins (non-MT-bound forms) [4].

In the present paper, the chemical speciation of biological trace metals using in-line hyphenated techniques is reviewed from the developmental standpoint, from HPLCAAS through HPLC/inductively coupled argon plasmaatomic emission spectrometry (ICP-AES) to HPLC-ICPmass spectrometry (MS). Methodological development and biological application are also focused on.

\section{Hyphenated techniques}

The quantification of metals became simpled with the introduction of spectroscopic methods, AAS and AES, and then $\mathrm{MS}$, in terms of measurement of multi-elements with high sensitivity with similar and simple procedures for the preparation of samples and determination of concentrations [2,3]. In addition, this spectrometric and mass spectrometric instrumentation made it possible to determine metal concentrations not only through off-line but also in-line connection with separation tools as element-specific detectors.

Biological constituents can be separated with diverse principles based on their various chemical and physicochemical properties, and the technical term "speciation" is defined by adapting the principle of separation [1]. The speciation of biological constituents at the chemical species level requires sophisticated procedures for separating the constituents into chemical species, at least depending on the chemical species of each metal, which can be performed most efficiently by means of various liquid chromatographic procedures, especially HPLC based on various separation principles [2,3]. It is now possible to use HPLC columns with diverse performance, such as gel filtration, ion exchange, affinity, reversed phase columns, etc. HPLC has shortened the time required for separation, reduced the amounts of samples, and increased the reproducibility (accuracy and precision), and further it has offered a new tool for speciation, namely, hyphenated techniques with the in-line connection with HPLC as a means of separation with AAS, ICP-AES or ICP-MS as an element-specific detector [2,3].

The separation of biological constituents on HPLC columns with diverse separation principles and in-line detec- 
tion with element-specific detectors, i.e. atomic absorption (AAS) and emission spectrometers (AES), or mass spectrometers (MS), have been developed as hyphenated techniques in the past 20 years. HPLC-AAS dramatically improved the process of speciation of biological metals, and reduced the time for analysis and the sample size. The use of AES with excitation with inductively coupled argon plasma (ICP) as the detector for HPLC, i.e. HPLC-ICP-AES, made it possible to speciate multi-elements simultaneously. Furthermore, the use of MS with ionization with ICP as the detector, i.e. HPLC-ICP-MS, has enabled the speciation of multi-elements with extremely high sensitivity, and at the same time it has made it possible to speciate not only endogenous but also exogenous metals with the use of enriched stable isotopes, making tracer experiments possible.

\section{HPLC-AAS}

Our first HPLC-AAS was successfully applied to separate metallothionein (MT) in biological samples [5]. The gel filtration column used for HPLC gave two distinct peaks for the two isoproteins of MT owing to the cation exchange property of the silica gel-based resin under weakly alkaline buffer conditions that are customarily employed to minimize the dissociation of metals from constituents. HPLC-AAS dramatically improved the analytical procedure for MT, that was not possible with other methods because of the absence of biological activities that can be used to quantitate MT [4].

The requirements for this hyphenated technique arise from the in-line connection of separation and detection tools in addition to the requirements for their single or separate use. Those required for the in-line connection, i.e. HPLCAAS, are i) adjustment of the difference between the flow rate on HPLC $(<1 \mathrm{~mL} / \mathrm{min})$ and the uptake rate of AAS $(4-5 \mathrm{~mL} / \mathrm{min})$, ii) a decrease in the concentrations of elements that produce inorganic salts on burning in the flame, and iii) a decrease in the buffer concentration so as not to disturb the nebulization, to prevent stacking the burner head, and so on. The first problem has been solved simply by introducing the eluate from the HPLC to the nebulizer of the AAS through a thin connecting tube, the difference in the flow rate being compensated for by the back pressure. The second and third problems can be solved by choosing appropriate buffers, for example, a low buffer concentration without or less $\mathrm{NaCl}$.

The dissociation of metals during separation procedures is of much concern for any analytical method for the separation of metals. The dissociation and redistribution of metals may take place depending on the presence of stronger ligands, i.e., functional groups, to metals such as buffers and resins. Silica gel-based resins certainly affect the distribution of metals bound rather loosely to constituents by redistributing them to the silanol and residual carboxyl groups in resins under alkaline buffer conditions. Metals may be redistributed from constituents to resins, and then from the resins to the constituents, resulting in distribution profiles of metals different from the native ones. The redistribution or randomization of metals is often observed between plasma proteins such as albumin and metals such as zinc, cadmium, copper and so on. Metals bound to enzymes are, in general, stable with usual separation procedures, i.e., stable in a weakly alkaline buffer solution.
The use of AAS as an element-specific detector for HPLC has made it possible to reduce the sample size and the time for analysis, and dramatically improved the reproducibility and detectability for MT. However, it can detect only a single metal in each run. Simultaneous detection of multi-elements is not possible by means of AAS.

\section{HPLC-ICP-AES}

AES with excitation with ICP can be used as a multi-element specific detector for HPLC. Vacuum ultraviolet ICPAES has been used as a detector and applied to speciate light elements such as sulfur and phosphorus, and also carbon, in addition to heavy metals in biological constituents, which made it possible to determine the elemental compositions of constituents (elemental analysis) by means of a single analytical procedure [6]. Although other limitations caused by the interference with elemental emission such as phosphorus emission have to be overcome for the use of ICP-AES as a detector, enormous benefit has been brought about by the use of ICP-AES instead of AAS. Namely, ICP-AES has made it possible to detect multi-elements simultaneously, and at the same time a wider range of elements, from light to heavy metals. These abilities of ICP-AES have been applied to speciate multi-elements in biological samples simultaneously, which has made it far easier to elucidate the mechanisms underlying biological interactions among endogenous multi-elements.

Figure 1 demonstrates the usefulness of HPLC-ICP-AES for the speciation of multi-elements in plasma. Many elements in different chemical forms can be speciated by a single analytical procedure with a small sample size of $0.1 \mathrm{~mL}$ in a short analytical time of less than $30 \mathrm{~min}$ with high reproducibility. The binding and distribution of metals injected into the bloodstream can be traced easily by speciation of each metal bound to plasma proteins. The distributions of metals and elements in milk serum determined by HPLC-ICP-AES show the dramatic changes in the concentrations and distributions of metals and elements with the number of days after parturition, especially in colostrum milk, which then become constant and simple in transitional milk [7]. Figure 2 demonstrates the usefulness of HPLCICP-AES, especially for the light element, sulfur (S).

\section{HPLC-ICP-MS}

ICP-AES is superior to AAS for the simultaneous determination of multi-elements, and the detection of a wide range of elements and metals, especially light elements in the vacuum ultraviolet detection mode. However, the sensitivity for the detection of metals and elements is not sufficient to detect ultra-trace elements in biological samples. MS is much more sensitive as a tool for detecting elements than AAS and AES, and MS with the ionization of elements with ICP became available from the late 1980s to the early 1990 s, and it is becoming much more popular in the fields of biochemistry, nutrition, toxicology and so on. In addition to simultaneous ultra-trace analysis of multi-elements, MS has shown a new applicability in tracer experiments, i.e. 


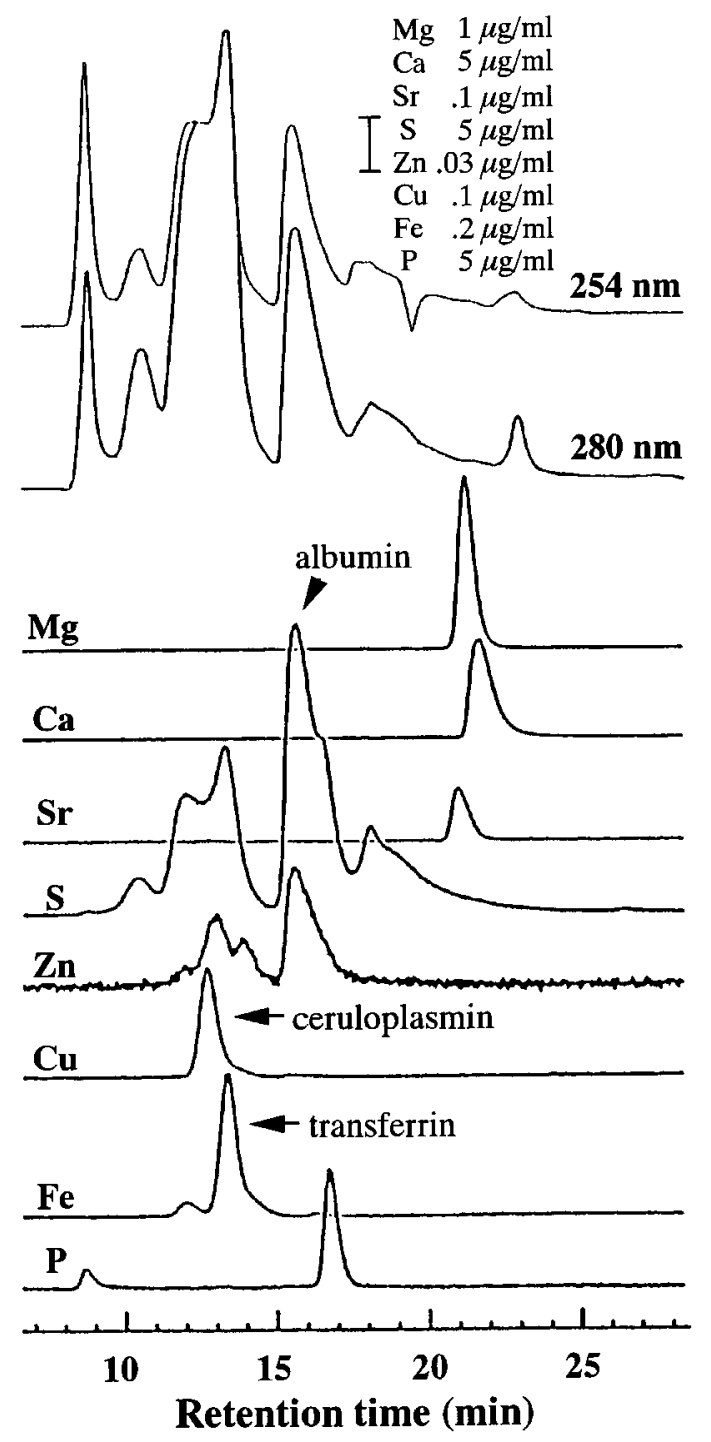

Figure 1. Distributions of elements in rat serum on a gel filtration column with the HPLC-ICP-AES method. A $0.1 \mathrm{~mL}$ portion of rat serum was applied to a GS 520 column $(7.6 \times 500 \mathrm{~mm}$, Showa Denko, Tokyo, Japan), and then the column was eluted with a $50 \mathrm{mM}$ Tris- $\mathrm{HCl}$ buffer solution, $\mathrm{pH} 7.4$, containing $0.1 \mathrm{M}$ $\mathrm{NaCl}$ at the flow rate of $0.1 \mathrm{~mL} / \mathrm{min}$. The eluate was introduced directly into an ICP-AES (JY48PVH; Seiko Instruments \& Electronics, Tokyo, Japan). The vertical bar indicates the detection level of each element with the off-line batch method.

enriched stable isotopes can be used as tracers, and the speciation of the tracers with hyphenated techniques has made it possible to carry out detailed metabolic and mechanistic studies that cannot be performed with radio-labeled isotopes and other techniques [8].

The use of enriched stable isotopes enhances the sensitivity for detecting elements with MS, due to the enrichment over the natural abundance. It also makes it possible to trace enriched (exogenous) elements together with endogenous elements, i.e. the speciation of both exogenous and endogenous multi-elements can be performed with a single procedure. A typical application is shown in figure 3, where
HPLC-ICP-MS was applied to reveal the mechanisms underlying the interaction between mercury $(\mathrm{Hg})$, selenium $(\mathrm{Se})$, and a plasma protein in the body [9].

$\mathrm{Hg}$ and $\mathrm{Se}$ are known to interact in the body and to reduce the toxicity of each other when both elements are administered simultaneously. The mechanism underlying this interaction in the bloodstream has been studied by intravenously injecting equimolar amounts of mercuric chloride and selenite into rats. Exogenous Se administered as $\left[{ }^{82} \mathrm{Se}\right]$-enriched selenite is taken up by and reduced in red blood cells (RBCs), and then transported into the plasma, where the reduced form of $\mathrm{Se}$ forms a unit complex $(\mathrm{Hg}-\mathrm{Se})_{n}$ transported with $\mathrm{Hg}$. The $(\mathrm{Hg}-\mathrm{Se})_{n}$ complex binds selectively to a plasma Se-containing protein, selenoprotein $\mathrm{P}$ (Sel P), to form a $(\mathrm{Hg}-\mathrm{Se})-\mathrm{Sel} \mathrm{P}$ complex. Sel $\mathrm{P}$ can be detected from natural abundance $\mathrm{Se}$ as an endogenous $\mathrm{Se}$. The $\mathrm{Hg} /{ }^{22} \mathrm{Se}$ ratio indicates the ratio of $\mathrm{Hg}$ to $\mathrm{Se}$ bound to $\mathrm{Sel} \mathrm{P}$, while the ratio of enriched and natural abundance $\mathrm{Se}\left({ }^{82} \mathrm{Se} /{ }^{77} \mathrm{Se}\right)$ gives the ratio of $\mathrm{Hg}$ and $\mathrm{Se}$ bound to Sel P. Data obtained on HPLC-ICP-MS suggested the formation of a $\{(\mathrm{Hg}-$ $\left.\mathrm{Se})_{n}\right\}_{m}-$ Sel P complex ( $m$ is the number of binding sites on Sel P) in the bloodstream, which explains the interaction to reduce the toxicity of $\mathrm{Hg}$ and $\mathrm{Se}$ each other. This speciation by HPLC-ICP-MS typically demonstrates the usefulness of MS as a detector for this hyphenated technique.

\section{Future of speciation studies with hyphenated techniques}

The chemical speciation of biological metals can be accomplished efficiently if the biological constituents in question can be separated from other constituents containing the same kinds of metals, and then the metals can be analyzed simultaneously with high sensitivity. The tools used for the separation of biological constituents have to be optimized for the best separation with the use of the best HPLC columns with diverse separation principles. SDS-PAGE is routinely used for biochemical separation. However, metals separated by this method cannot be detected directly by ICP-MS. It will be much more convenient if one of the most popular detection methods in biochemistry can be used for speciation or HPLC columns that are comparable to those for SDS-PAGE become available.

Tools for the detection of metals have been developed, from AAS, AES to MS. This detection instrumentation requires that metals and elements are excited or ionized as atoms, whereon important information as to ligands (binding proteins) for metals and elements is lost, which can be prevented if the metals are detected as metal-binding proteins, for example, by electrospray ionization (ESI) or matrix-assisted laser desorption ionization-time of flight (MALDI-TOF) mass spectrometry.

Among biological constituents that participate in the handling of metals, metalloenzymes have been studied extensively because of the easy detection and speciation with their biological activities. However, proteins that bind to metals for transfer or storage have only been studied to a limited extent because of a lack of easy detection methods other than separation and detection as metal-binding proteins. 


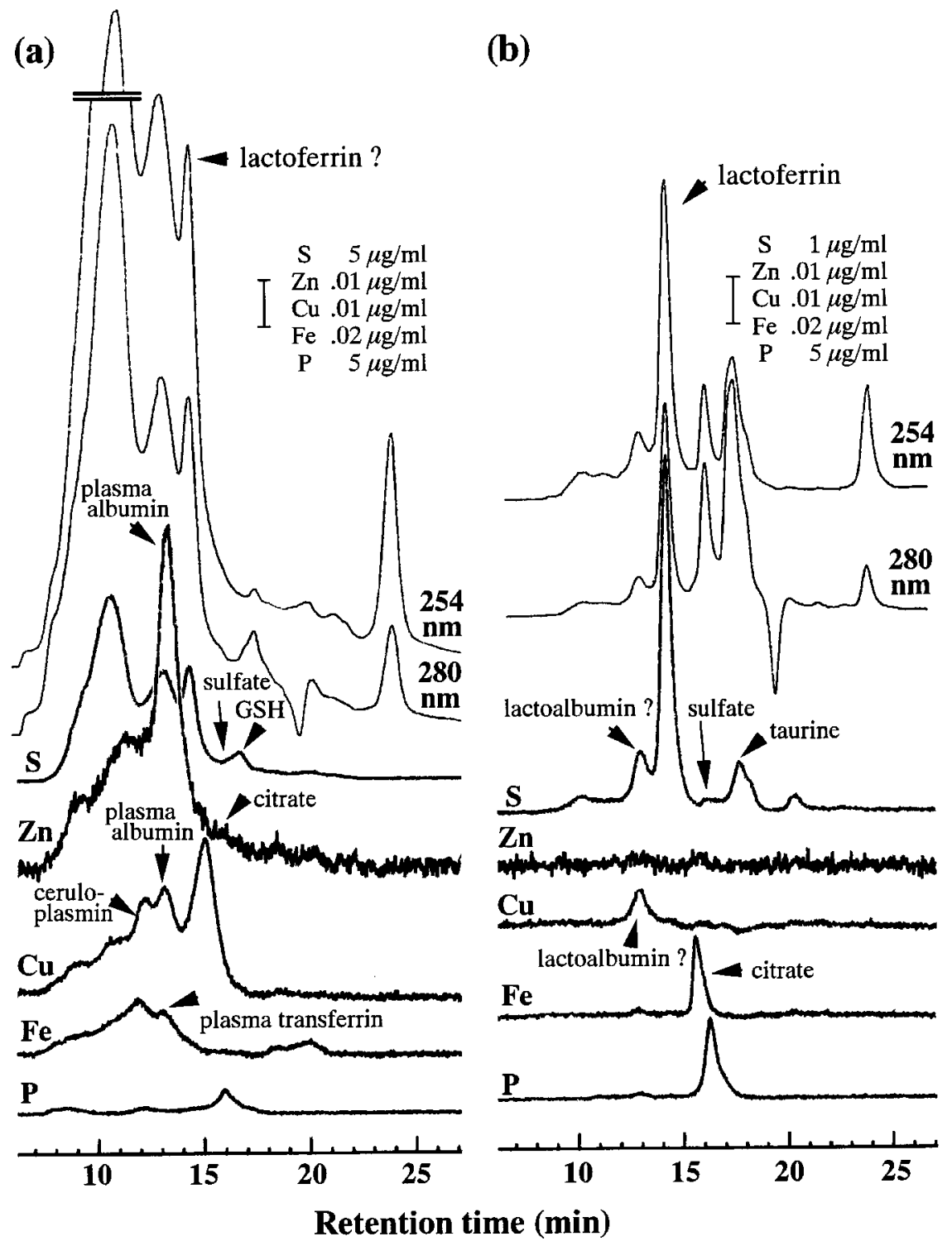

Figure 2. Distributions of elements in breast milk serum on a gel filtration column with the HPLC-ICP-AES method. A $0.1 \mathrm{~mL}$ portion of the serum of breast milk on days 2 a) and 31 b) of parturition was analyzed as described in the legend to figure 1 [7].
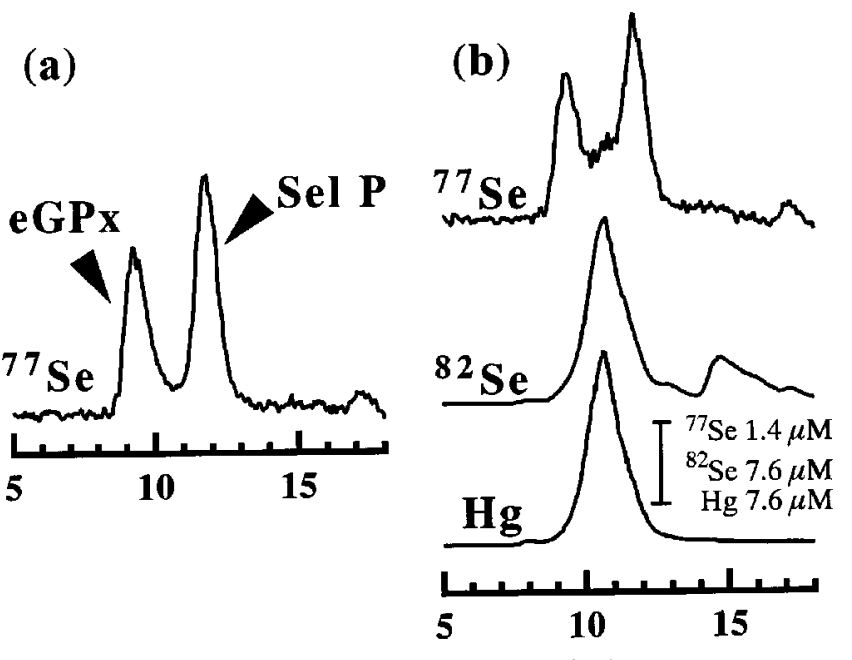

Retention time (min)
These metal-binding proteins are some of the most favorable targets for the hyphenated techniques, and the speciation of these metal-binding proteins can be accomplished most easily by means of the hyphenated techniques. It has been demonstrated that metal-binding proteins belonging to this category function in the regulation of copper $(\mathrm{Cu})$.

Figure 3. Distributions of $\mathrm{Hg}$, and endogenous and exogenous $\mathrm{Se}$ in serum after intravenous co-injection of equimolar amounts of mercuric chloride and selenite into rats. Equimolar amounts of [ $\left.{ }^{22} \mathrm{Se}\right]-e n r i c h e d$ selenite and natural abundance mercuric chloride were injected intravenously into a Wistar rat at the dose of $4 \mu \mathrm{mol} / \mathrm{kg}$ body weight. A $0.1 \mathrm{~mL}$ portion of serum prepared from blood obtained $10 \mathrm{~min}$ after the injection was analyzed by the HPLC-ICP-MS method on a GS 520 column with detection with ICP-MS (HP4500; Yokogawa Analytical Systems, Musashino, Japan). 
$\mathrm{Cu}$-binding P-type ATPase for the efflux of $\mathrm{Cu}$ plays a central role in $\mathrm{Cu}$ homeostasis, and its mutation on the X-chromosome and autosome 13 causes Menkes disease and Wilson disease, respectively [10]. More recently, several $\mathrm{Cu}$ chaperones have been demonstrated to transport $\mathrm{Cu}$ specifically to intracellular organelles and proteins such as mitochondria [11] and $\mathrm{Cu}, \mathrm{Zn}$-superoxide dismutase (SOD), respectively [12]. The presence of these proteins was first proposed based on the corresponding gene structures. However, if hyphenated techniques are sensitive enough to detect $\mathrm{Cu}$ bound to chaperones, these proteins should be speciated as metal-binding proteins. MT is the only metal-binding protein in a cell that has been routinely speciated as a metal-binding protein having no biological activity that can be used for detection. Albumin has been recognized as the major metal-binding protein that participates in the transport of metals outside cells, i.e. an extracellular chaperone. In addition to these major metal-binding proteins, intracellular and extracellular chaperones that exist in small quantities should be the next target for the hyphenated techniques.

\section{References}

1. The First International Conference on Trace Element Speciation in Biomedical, Nutritional and Environmental Sciences, Neuherberg, Germany, May, 1998.

2. Karjalainen, E. J.; Karjalainen, U. P. Eds., Data Analysis for Hyphenated Techniques, Elsevier, Amsterdam, 1996; pp 475.

3. Lobinski, R.; Marczenko, Z. Spectrochemical Trace Analysis for Metals and Metalloids, Elsevier, Amsterdam, 1996; pp 808.

4. Webb, M. Experientia Suppl. 1987, 52, 109-134.

5. Suzuki, K. T. Anal. Biochem. 1980, 102, 31-34.

6. Morita, M.; Uehiro, T.; Fuwa, K. Anal. Chem. 1980, 52, 349351.

7. Suzuki, K. T.; Tamagawa, H.; Hirano, S.; Kobayashi, E.; Takahashi, K. Shimojo, N. Biol. Trace Elem. Res. 1991, 28, 109-121.

8. Suzuki, K. T. Tohoku J. Exp. Med. 1996, 178, 27-35.

9. Yoneda, S.; Suzuki, K. T. Biochem. Biophys. Res. Commun. 1997, 231, 7-11.

10. Monaco, A. P.; Chelly, J. Adv. Genet. 1995, 33, 233-253.

11. Amaravadi, R.; Glerum, D. M.; Tzagoloff, A. Hum. Genet. 1997, 99, 329-333.

12. Culotta, V.C.; Klomp, L.W.; Strain, J., Casareno, R.L.; Krems B.; Gitlin, J.D. J. Biol. Chem. 1997, 272, 23469-23472. 